\title{
Efficacy of visual process improvement in patients with homonymous hemianopia
}

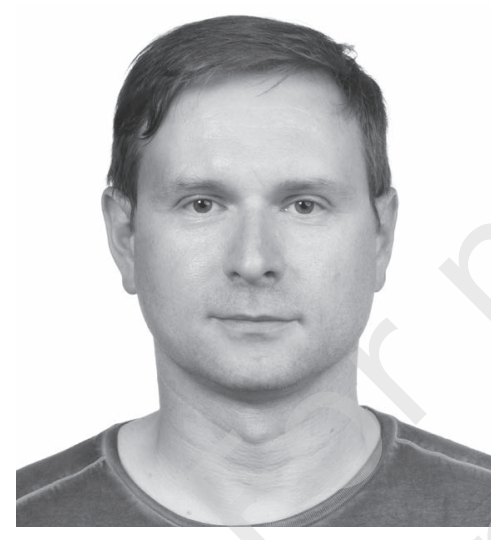

Sławomir Nogaj ${ }^{13}$, Katarzyna Dubas', Andrzej Michalski

'Department of Optometry, Chair of Ophthalmology and Optometry Poznan University of Medical Sciences, Poznan, Poland Head: Prof. Wojciech Warchoł, PhD ${ }^{2}$ Department of Ophthalmology, Chair of Ophthalmology and Optometry, Poznan University of Medical Sciences, Poznan, Poland Head: Prof. Marcin Stopa, MD, PhD, FEBO Laboratory of Vision Science and Optometry, Faculty of Physics, Adam Mickiewicz University, Poznan, Poland Head: Prof. Ryszard Naskręcki, PhD

H I G H LI GHTS

Improvement of the visual functions in patients with homonymous hemianopia is possible by applying prism correction and visual rehabilitation.

\section{ABSTRACT}

Homonymous hemianopia is a visual field defect following a stroke, tumor development, traumatic brain injury and other rare causes. Due to the damage to the visual system, lateral neglect occurs that induces difficulties with detecting objects on one side of the binocular visual field, which exposes patients to potential danger during daily activities, especially performed outside - in traffic. Different optical aids such as Fresnel prism have been developed to help those patients by increasing the perception of the affected hemifield. This prism correction can appear in various configurations, for instance: yoked prism, monocular and binocular sector prisms placed with a base to the affected side. The other option to deal with these patients is visual training based on stimulating the affected hemifield.

Key words: homonymous hemianopia, loss of visual field, prism correction 


\section{INTRODUCTION}

Homonymous hemianopia $(\mathrm{HH})$ is a loss of the two right or two left halves of both eyes' visual fields. It is caused by damage to the visual pathway beyond the optic chiasm. Characteristics of the visual field defect and the accompanying neurological symptoms are used to determine the location of pathological changes in the brain [1]. This type of defect is most often the result of a stroke (in nearly $70 \%$ of cases), trauma, tumor, surgery, or other, less frequent causes [2]. Self-improvement of the field of view is possible shortly after the brain injury. Zhang et al. observed improvement in over $38 \%$ of patients, usually within three weeks from the onset of the ischemic stroke event. Self-improvement of the visual field defect is unlikely after 6 months after brain injury [3]. Therapeutic and rehabilitation methods improving the functioning of patients with $\mathrm{HH}$ are rarely used because their effectiveness is not well documented in every case. They can be divided into passive, the purpose of which is to use the intact part of the field of view using prisms, and active, i.e. visual training focused on facilitating adaptation to the state of amblyopia and stimulating the area of the visual field covered by the defect $[4,5]$. Patients with $\mathrm{HH}$ have significant reading difficulties because they cannot see the letters following the readings on the right side. When the defect extends to the left side, it is difficult to move to the next text line in the visual field affected by the visual impairment [6]. There are reports showing improvement of these visual functions after introducing training to improve saccades [7]. The partial loss of the visual field also means impairing the driving ability, significantly reducing road safety [8].

\section{APPLICATION OF PRISMATIC CORRECTION}

In the case of visual field defects, it possible to use a press-on prism in a selected area of an eyeglass lens with the base facing the defect and the edge near the central field of view. The use of prisms shifts the image closer to the center of the field of view, reducing the need for a head adjustment movement. In the case of damage to the visual pathway beyond the optic chiasm, we can deal with the $\mathrm{HH}$ opposite to the damaging side. If the defect affects the right sides of the visual fields, it is possible to use prisms on both eyeglass lenses' right parts. In this case, the base of the used prisms is directed to the right, which shifts the field of view covered by the defect to the patient's area when looking right. In the past, binocular prisms were usually used in the areas affected by the defect. Later it was practiced to position the Fresnel prism in front of only one eye, where the defect occurs from the temporal side (fig. 1). Although this type of optical aid improves patients' functioning with hemianopia, it has the limitation, the most troublesome of which is diplopia close to the central field of vision, which is unacceptable for many patients $[9,10]$. Another solution was to use sector prisms of high power (30-40 prism diopters) proposed by Peli [11, 12]. Usually, two prismatic segments with the base out orientation are placed on the eyeglass lens: one at the height of the top and the other at the bottom of the corneal limbus. This solution, which causes peripheral diplopia, allows the visual field area to be widened by approx. $20^{\circ}$ (fig. 2).

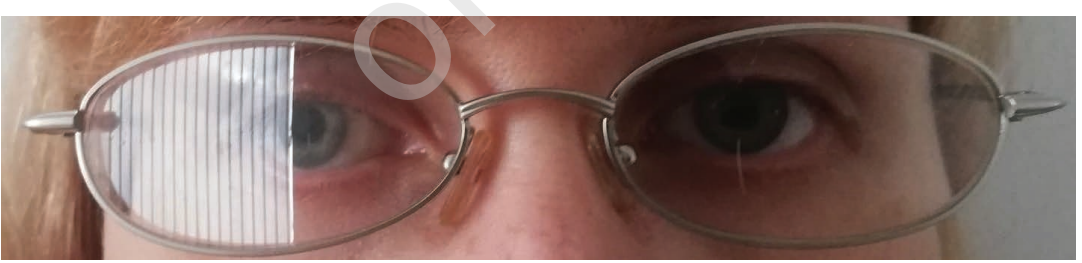

\section{FIGURE 2}

\section{Application of sector prisms, according to Eli Peli [K. Dubas].}

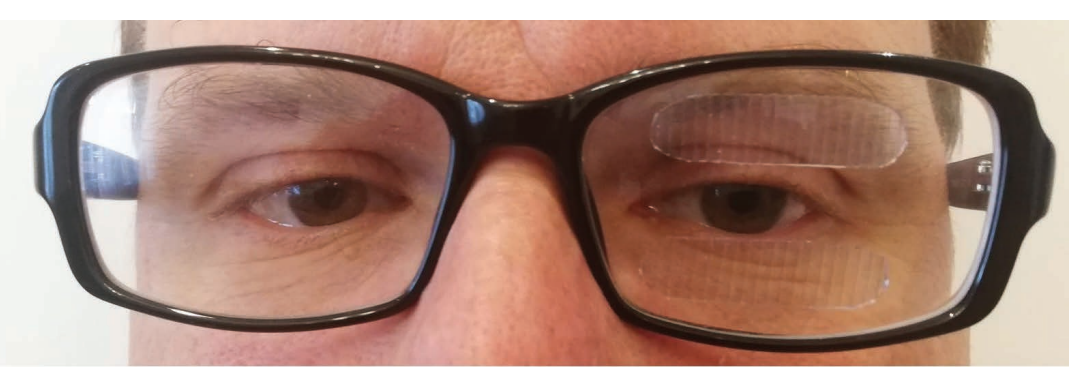




\section{ACTIVE METHODS OF IMPROVING VISUAL FUNCTIONS}

Brain neuroplasticity is the nervous system's ability to regenerate and create new connections to adapt and reorganize in response to environmental changes. Studies in humans, monkeys, and other mammals have shown that the regeneration of axons and dendritic cells can occur in adults if they learn new things and gain experience and training [13-15]. This reason is essential to support and improve these processes through rehabilitation [16].

The Kasten and Sabel research group has designed a computer software called Vision Restoration Training (VRT) that can be adapted to the patient's hemianopia disorders visual stimuli are presented on the border between the narrowed and normal field of vision at the same time. The patient's task is to fix his eyesight to the central point. In this way, the neurons remaining in the ischemic zone of the brain are activated. Training can be done in a home environment on a computer (PC) and lasts an hour, and the whole program is prepared for 6 months. In the experiment, an improvement in the field of view by $5^{\circ}$ was observed in $95 \%$ of patients participating in the training [17]. However, these results were questioned by the Pollock Group - the VRT developers were accused of inaccurate assessment of the improvement in the field of vision because, according to critics, the use of the VRT program did not allow for a precise assessment of eye fixation [18]. Kasten's research group repeated their research with a changed methodology a microperimetry and a scanning laser ophthalmoscope were used [19]. In this experiment, the authors did not notice any significant changes in the visual field. Instead, they found that it was due to improved eye movements. Even though the VRT method does not significantly affect widening the field of vision, many patients report an improvement in daily activities after training.

Studies by Julkunen et al. showed changes in visual evoked potentials and functional magnetic resonance imaging after training with the VRT program [20].

The scientific paper by Zihl presents an analysis of oculomotor aspects in 60 patients with $\mathrm{HH}$ and a preserved central field of view smaller than $5^{\circ}$. The author found that the subgroup of patients who underwent visual rehabilitation (saccades) in the final studies showed a significant improvement in visual search speed thanks to the improvement of oculomotor adaptation [21].

In the studies by Roth et al., stimulation in the area of the disturbing visual field by flicker stimulation training (FT) was compared with explorative saccade training (EST) [22]. Researchers saw significant improvements in the accuracy and speed of visual tasks in the group after saccade training. On the other hand, the subjects after the flicker training did not improve visual tasks. There were no visual field changes in any of the groups. Visual perception deficits caused by damage to the parietal lobe almost completely disappear after training, confirmed by Kerkhoff [23]. Patients after rehabilitation showed better orientation and location of objects in space.

\section{CONCLUSIONS}

- HH means a loss of visual field in the left or right field of vision in both eyes.

- HH most often has a stroke etiology; other causes are traumatic injuries, tumors, and other structural brain disorders.

- The specific features of the visual field defect and other neurological symptoms help locate the lesion.

- In most cases, spontaneous improvement of HH is possible within three months after the onset of the visual impairment. Self-improvement of the visual field defect is unlikely after 6 months after brain injury.

- Management options are limited and include optical widening/shifting of the visual field and enhancement techniques for improving visual functions.

$\mathrm{HH}$ is a condition that significantly limits the functioning of the patient in everyday life. Properly selected prismatic correction is an effective method of helping patients and can be quickly implemented. Visual training takes longer to achieve any improvement.

Figures: from the author's own materials.

\section{CORRESPONDENCE}

\section{Sławomir Nogaj, MSC}

Laboratory of Vision Science and Optometry, Faculty of

Physics, Adam Mickiewicz University, Poznan, Poland

61-614 Poznan, Uniwersytetu Poznańskiego 2

phone: (61) 8295188

e-mail: snogaj@amu.edu.pl

\section{ORCID}

Sławomir Nogaj - ID - http://orcid.org/0000-0001-5306-7952

Katarzyna Dubas - ID - http://orcid.org/0000-0003-2443-7285

Andrzej Michalski - ID - http://orcid.org/0000-0003-0260-1320 


\section{References}

1. Trobe JD. Visual fields. In: Trobe JD (ed). The Neurology of Vision. Oxford, Oxford 2001: 109.

2. Zhang X, Kedar S, Lynn MJ et al. Homonymous hemianopias: clinical-anatomic correlations in 904 cases. Neurology. 2006; 66(6): 906-10.

3. Zhang X, Kedar S, Lynn MJ et al. Natural history of homonymous hemianopia. Neurology. 2006; 66(6): 901-5.

4. Bowers AR, Keeney K, Peli E. Community-based trial of a peripheral prism visual field expansion device for hemianopia. Arch Ophthalmol. 2008; 126(5): 657-64.

5. Pambakian AL, Kennard C. Can visual function be restored in patients with homonymous hemianopia? Br J Ophthalmol. 1997; 81(4): 324-8.

6. Schuett S, Heywood CA, Kentridge RW et al. Rehabilitation of hemianopic dyslexia: are words necessary for re-learning oculomotor control? Brain. 2008; 131(12): 3156-68.

7. Pambakian AL, Mannan SK, Hodgson TL et al. Saccadic visual search training: a treatment for patients with homonymous hemianopia. J Neurol Neurosurg Psychiatry. 2004; 75(10): 1443-8.

8. Racette L, Casson EJ. The impact of visual field loss on driving performance: evidence from on-road driving assessments. Optom Vis Sci. 2005; 82(8): 668-74.

9. Lee AG, Perez AM. Improving awareness of peripheral visual field using sectorial prism. J Am Optom Assoc. 1999; 70(10): 624-8.

10. Szlyk JP, Seiple W, Stelmack J et al. Use of prisms for navigation and driving in hemianopic patients. Ophthalmic Physiol Opt. 2005; 25(2): 128-35.

11. Peli E. Field expansion for homonymous hemianopia by optically induced peripheral exotropia. Optom Vis Sci. 2000; 77(9): $453-64$.

12. Peli E. Vision multiplexing: an engineering approach to vision rehabilitation device development. Optom Vis Sci. 2001; 78(5): $304-15$.

13. Balliet R, Blood KM, Bach-y-Rita P. Visual field rehabilitation in the cortically blind? Neurol Neurosurg Psychiatry. 1985; 48(11): 1113-24.

14. Marsálek P, Santamaría F. Investigating spike backpropagation induced Ca2+ influx in models of hippocampal and cortical pyramidal neurons. Biosystems. 1998; 48(1-3): 147-56.

15. Macias M. Injury induced dendritic plasticity in the mature central nervous system. Acta Neurobiol Exp (Wars). 2008; 68(2): 334-46.

16. Grunda T, Marsalek P, Sykorova P. Homonymous hemianopia and related visual defects: Restoration of vision after a stroke. Acta Neurobiol Exp (Wars). 2013; 73(2): 237-49.

17. Kasten E, Sabel BA. Visual field enlargement after computer training in brain-damaged patients with homonymous deficits: an open pilot trial. Restor Neurol Neurosci. 1995; 8(3): 113-27.

18. Pollock A, Hazelton C, Henderson CA et al. Interventions for visual field defects in patients with stroke. Cochrane Database Syst Rev. 2011; (10): CD008388.

19. Kasten $E$, Guenther T, Sabel BA. Inverse stimuli in perimetric performance reveal larger visual field defects: implications for vision restoration. Restor Neurol Neurosci. 2008; 26(4-5): 355-64.

20. Julkunen L, Tenovuo O, Jääskeläinen $S$ et al. Rehabilitation of chronic post-stroke visual field defect with computer-assisted training: a clinical and neurophysiological study. Restor Neurol Neurosci. 2003; 21(1-2): 19-28.

21. Zihl J. Visual scanning behavior in patients with homonymous hemianopia. Neuropsychologia. 1995; 33(3): $287-303$.

22. Roth T, Sokolov AN, Messias A et al. Comparing explorative saccade and flicker training in hemianopia: a randomized controlled study. Neurology. 2009; 72(4): 324-31.

23. Kerkhoff G. Neurovisual rehabilitation: recent developments and future directions. J Neurol Neurosurg Psychiatry. 2000; 68(6): 691-706. 
Efficacy of visual process improvement in patients with homonymous hemianopia

S. Nogaj, K. Dubas, A. Michalski

\section{Authors' contributions:}

Sławomir Nogaj: concepts and design, manuscript preparation, literature search; Katarzyna Dubas: manuscript preparation and review, literature search; Andrzej Michalski: manuscript preparation and review, literature search.

\section{Conflict of interest:}

None.

\section{Financial support:}

None.

\section{Ethics:}

The content presented in the article complies with the principles of the Helsinki Declaration, EU directives and harmonized requirements for biomedical journals. 